\title{
Uso da Fabricação Digital para o Design de Superfície em Produtos de Moda
}

\section{Use of Digital Manufacturing for Surface Design in Fashion Products}

\author{
lana Uliana Perez \\ Mestranda, Universidade Federal do Paraná, PR \\ ianauliana@hotmail.com \\ Aguinaldo dos Santos \\ Doutor, Universidade Federal do Paraná, PR \\ asantos@ufpr.br
}




\title{
Uso da Fabricação Digital para o Design de Superfície em Produtos de Moda
}

Use of Digital Manufacturing for Surface Design in Fashion Products

lana Uliana Perez, Aguinaldo dos Santos

\section{Resumo}

O presente trabalho apresenta as possibilidades de uso de tecnologias de fabricação digital no Design de Superfície em produtos de moda, demonstrando como essas tecnologias podem contribuir para o envolvimento do usuário nos processos de criação e de produção. Mais especificamente, o foco deste artigo são os usos da fabricação digital para a personalização de produtos e para a fabricação pessoal, em um contexto de produção distribuída. O método de pesquisa adotado é a revisão bibliográfica assistemática e sistemática, tendo sido consultadas três bases de dados, repositórios online de sete periódicos nacionais, assim como livros, artigos publicados em anais de eventos, sites e blogs especializados. Como resultado, são apresentados alguns exemplos de uso de tecnologias de fabricação digital no Design de Superfície de produtos de moda, discutindo as possibilidades e limitações das tecnologias atuais para o fomento de um cenário de produção distribuída.

Palavras-chave: design de superfície, produção distribuída, fabricação pessoal

\begin{abstract}
This paper presents the possibilities of applying digital manufacturing technologies for Surface Design in fashion products, demonstrating how these technologies may contribute to the user's involvement in creation and production processes. More specifically, the focus of this article is on the uses of digital manufacturing for product personalization and for personal fabrication in a context of distributed production. The research method adopted is systematic and unsystematic bibliographic review. We consulted three databases and seven national journals repositories, as well as books, articles published in conference proceedings, specialized websites, and blogs. Therefore, the article presents some examples of digital manufacturing technologies application in fashion products Surface Design, discussing the possibilities and limitations of current technologies for the promotion of a distributed production scenario.
\end{abstract}

Keywords: surface design, distributed production, personal fabrication 


\section{Introdução}

O intuito do presente artigo é mapear o emprego da fabricação digital em Design de Superfície na área de moda, tendo como escopo as tecnologias pertinentes para a personalização de produtos e para a fabricação pessoal. A partir dos exemplos de aplicação encontrados na literatura, demonstra-se como a fabricação digital e o próprio Design de Superfície podem contribuir para o envolvimento do usuário nos processos de criação e de produção.

Cabe ressaltar que este artigo tem como enfoque as possibilidades apresentadas pela fabricação digital para a criação e produção de artigos de moda em um modelo de produção distribuída, entendida neste trabalho como "unidade produtiva de pequena escala, no próprio local de uso ou perto deste, na qual os usuários são os produtores - sejam indivíduos, pequenos negócios e/ou uma comunidade local" (LeNSin, 2016, p. 7, tradução nossa). Não compete a este artigo, portanto, discutir a aplicação em larga escala das tecnologias de fabricação digital aqui apresentadas.

Ademais, considerando que as ferramentas de fabricação digital ainda estão em fase de desenvolvimento, conforme atesta Gershenfeld (2012), o presente artigo não intenciona aprofundar-se nos aspectos técnicos das tecnologias apresentadas, pois as atualmente disponíveis para o setor de vestuário podem, em médio prazo, ser aprimoradas ou substituídas por novas tecnologias de fabricação digital. Desse modo, o presente trabalho atém-se a discutir as possibilidades de uso da fabricação digital para o Design de Superfície em produtos de moda.

\section{Fabricação digital e produção distribuída}

O termo "fabricação digital" refere-se a processos que utilizam máquinas controladas por computador, isto é, máquinas de comando numérico computadorizado (CNC) capazes de interpretar arquivos de $C A D^{1}$, traduzindo-os em coordenadas para a fabricação do objeto, seja por adição ou subtração de material. (Gershenfeld, 2012; Neves, 2014).

As tecnologias de fabricação digital começaram a se tornar populares no início dos anos 2000, devido ao desenvolvimento de projetos open hardware ${ }^{2}$, que difundiram o seu uso ao compartilhar online projetos que podem ser modificados e 
melhorados de modo colaborativo. Os interessados passaram a poder construir suas próprias máquinas de fabricação digital em casa, a partir dos projetos compartilhados online e da aquisição de kits de montagem (Anderson, 2012; Bauwens Et Al., 2012; Gershenfeld, 2012).

Com a introdução de tecnologias open hardware e a expansão de comunidades para compartilhamento dos equipamentos (os makerspaces), a fabricação digital tornou-se mais acessível. Desse modo, começou a ser utilizada também para a fabricação pessoal. Isso revelou o seu potencial para a produção local sob demanda e para a personalização dos produtos, combinando tecnologia com produção artesanal (Balka; Raasch; Herstatt, 2009; Neves; Rossi, 2011; Troxler, 2011; Anderson, 2012; Gershenfeld, 2012).

O potencial uso das tecnologias de fabricação digital para a produção sob demanda, seja por meio da personalização de produtos ou pela fabricação pessoal, faz com que essas tecnologias se enquadrem em um cenário de produção distribuída. O conceito de produção distribuída inclui tanto práticas atuais quanto emergentes que aumentam a capacidade dos usuários de intervir no design e na produção dos artefatos que adquirem (Kohtala, 2015). Isso implica "mudança nos padrões de consumo e de produção, distanciando-se da produção em massa convencional, com suas cadeias de suprimentos longas e lineares, economias de escala e tendências centralizadoras" (Ibidem, p. 654, tradução nossa).

A inserção de tecnologias de fabricação digital na indústria de moda representa, portanto, uma alternativa à produção em massa e aos produtos padronizados do fast fashion, pois viabiliza a personalização da produção (Richardson, 2015; Bruno, 2016). Ademais, sua adoção em um cenário de produção distribuída é importante para que a indústria de moda se antecipe às tendências identificadas para a indústria manufatureira.

Segundo estudo promovido pela $\mathrm{ABIT}^{3}$, pela $\mathrm{ABDI}^{4}$ e pelo $\mathrm{SENAI}$ $\mathrm{CETIQT}^{5}$, algumas das principais características da futura indústria de moda, nomeada Confecção 4.0, alinham-se ao conceito de produção distribuída: aproximação entre produtor e consumidor final; difusão de tecnologias sustentáveis; virtualização da produção; disseminação de mini fábricas automatizadas; 
personalização dos produtos (Bruno; Pimentel, 2017; Abdi; Fundação Certi; 2015; Bruno, 2016).

O estudo ressalta que a indústria de vestuário, tradicionalmente identificada como de baixa intensidade tecnológica, sofrerá um processo de intensificação do uso de tecnologias, com a adoção, dentre outras, das tecnologias de fabricação digital (Ibidem). Os designers que atuam na indústria da moda devem, portanto, seguir a recomendação do Centro Brasil Design de reconhecer e incorporar inovações tecnológicas, tendo em vista antecipar-se ao cenário futuro e vislumbrar novas possibilidades para o seu trabalho (CBD, 2014).

Contudo, pesquisa realizada por Bastos (2014, p. 123) revela que "os profissionais da área da moda [atualmente] desconhecem as possibilidades da fabricação digital", embora demostrem interesse ou curiosidade. Por isso, a pesquisadora supracitada ressalta a importância de evidenciar as oportunidades apresentadas pela fabricação digital, tornando o conhecimento mais acessível.

\section{Materiais e método}

A pesquisa realizada é qualitativa de caráter exploratório e de natureza aplicada. O método adotado é a Revisão Bibliográfica assistemática (RBA) e sistemática (RBS), um tipo de pesquisa bibliográfica que segue um método explícito, planejado e justificável (Dresch; Lacerda; Antunes Júnior, 2015). A RBS diferenciase da revisão assistemática por apresentar maior rigor científico, "podendo alcançar melhores resultados e reduzir erros e o viés do pesquisador responsável pela investigação" (Conforto; Amaral; Silva, 2011, p. 2).

A RBS foi conduzida com base no roteiro proposto por Conforto, Amaral e Silva (2011), que dividem a revisão em 15 etapas distribuídas em três fases, como demonstra a figura 1. As bases de dados consultadas foram a Biblioteca Digital Brasileira de Teses e Dissertações ${ }^{6}$, o Portal de Periódicos da Capes e o Google Scholar. O principal critério de inclusão adotado foi a data de publicação, que não deveria exceder 10 anos. Na consulta ao Portal de Periódicos da Capes, também foram acrescentados critérios relativos ao idioma, que deveria ser inglês, e ao tipo de material, restringindo a busca a artigos publicados em periódicos revisados pelos pares. Nas outras bases de dados, buscou-se por trabalhos publicados no Brasil. 

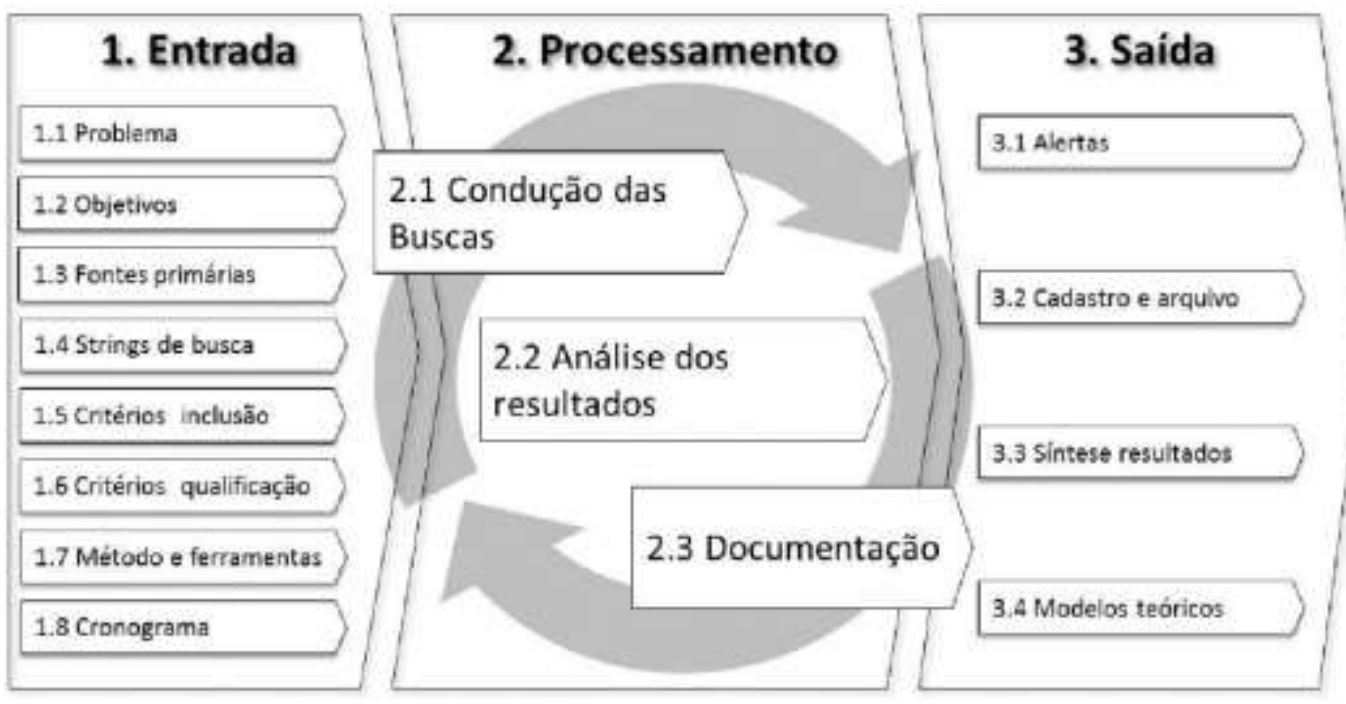

Figura 1: Roteiro para realização da RBS (Conforto, Amaral e Silva, 2011, p. 7)

Foram aplicados dois filtros para a seleção dos trabalhos. No primeiro, por meio da análise de título, resumo e palavras-chave, foram selecionadas publicações referentes à fabricação digital e à cadeia produtiva de vestuário. No segundo filtro, foram considerados método, resultados, discussão e considerações finais. As publicações foram selecionadas com base na qualidade do trabalho e na relevância para a pesquisa realizada. Os resultados das buscas são apresentados no quadro a seguir:

\begin{tabular}{|c|c|c|c|c|c|}
\hline Base de dados & Critérios de inclusão & String & Resultados & Filtro 1 & Filtro 2 \\
\hline \multirow{2}{*}{$\begin{array}{l}\text { Portal de } \\
\text { Periódicos da } \\
\text { Capes }\end{array}$} & \multirow{2}{*}{$\begin{array}{l}\text { Tipo de material: artigos } \\
\text { publicados em periódicos } \\
\text { revisados pelos pares } \\
\text { Idioma: inglês } \\
\text { Data de publicação: } \\
\text { últimos } 10 \text { anos } \\
(2007-2017)\end{array}$} & $\begin{array}{c}\text { (textile OR fashion OR } \\
\text { clothing) AND "surface } \\
\text { design" AND digital } \\
\text { (manufacturing OR } \\
\text { production) }\end{array}$ & 69 & 7 & 1 \\
\hline & & $\begin{array}{l}\text { (clothing OR fashion) } \\
\text { AND ("digital } \\
\text { manufacturing" OR } \\
\text { "digital fabrication") }\end{array}$ & 183 & 36 & 3 \\
\hline \multirow{2}{*}{$\begin{array}{l}\text { Google } \\
\text { Scholar }\end{array}$} & \multirow{2}{*}{$\begin{array}{l}\text { Data de publicação: } \\
\text { últimos } 10 \text { anos } \\
(2007-2017)\end{array}$} & $\begin{array}{l}\text { "design de superfície" } \\
\text { AND "fabricação digital" }\end{array}$ & 8 & 0 & 0 \\
\hline & & $\begin{array}{l}\text { "design de superfície" } \\
\text { AND "fabricação digital" } \\
\text { AND "moda OR têxtil" }\end{array}$ & 5 & 0 & 0 \\
\hline $\begin{array}{l}\text { Biblioteca Digital } \\
\text { Brasileira de } \\
\text { Teses e } \\
\text { Dissertações }\end{array}$ & $\begin{array}{l}\text { Data de publicação: } \\
\text { últimos } 10 \text { anos } \\
(2007-2017)\end{array}$ & $\begin{array}{c}\text { "fabricação digital” AND } \\
\text { design }\end{array}$ & 19 & 2 & 2 \\
\hline
\end{tabular}

Tabela 1: Resultados da RBS (A autora, 2017) 
Devido à pequena quantidade de trabalhos encontrados, foram também realizadas buscas em alguns dos principais periódicos nacionais de moda e de design: ModaPalavra, dObra[s], Iara, Estudos em Design, Redige, Strategic Design Research, Design \& Tecnologia e Tríades. Todas as buscas nos repositórios digitais dos periódicos consultados adotaram as mesmas palavras-chave: "fabricação digital" e "digital". Para a seleção dos artigos, foram aplicados os mesmos critérios de inclusão e filtros mencionados anteriormente. Ao final da RBS, foram selecionadas duas dissertações de mestrado (Bastos, 2014; Oliveira, 2013), quatro artigos internacionais (Kohtala, 2015; Akiwowo et al., 2014; Atwell, 2014; Niinimäki; Hassi, 2011) e cinco artigos nacionais (Carvalho; Rüthschilling, 2016; Souza; Reis, 2014; Guimarães, 2014; Neira, 2012; Cunha, 2011).

A partir das publicações selecionadas, foi efetuada busca cruzada, que consiste, segundo Conforto, Amaral e Silva (2011, p. 10), em rastrear trabalhos relevantes por meio das citações dos autores, tendo como objetivo "identificar estudos relevantes que não foram encontrados durante a busca nos periódicos ou bases de dados". Para complementar a bibliografia, também foi realizada revisão bibliográfica assistemática por meio da consulta a livros, teses e dissertações internacionais, artigos publicados em anais de congressos nacionais e internacionais, assim como publicações em sites e blogs especializados. Os resultados da revisão bibliográfica são apresentados a seguir, divididos de acordo com as principais tecnologias identificadas.

\section{Tecnologias de fabricação digital}

Para a fabricação digital de vestuário, Niinimäki e Hassi (2011) indicam máquinas de estamparia digital, bordadeiras digitais, cortadoras a laser e máquinas de tecelagem digital ${ }^{7}$. Bastos (2014) e Strien e Pont (2016) apontam, ainda, as impressoras 3D, enquanto Bastos (2014) apresenta a cortadora de vinil e a fresadora CNC como alternativas. São relatados a seguir todos os usos de tecnologias de fabricação digital identificados na literatura consultada que sejam oportunos ao Design de Superfície de produtos de moda e ao modelo de produção distribuída. 


\subsection{Impressoras 3D}

As impressoras 3D tem sido utilizadas, na área de moda, principalmente para a criação de materiais similares ao tecido (Velden, 2016). A primeira roupa impressa em 3D foi o Black Drape Dress, produzido em 2000 pelo engenheiro Jiri Evenhuis em colaboração com o designer holandês Janne Kyttanen (Kuhn; Minuzzi, 2015). Dez anos depois, o escritório de design Continuum também utilizou a impressão 3D, mas para a confecção de um biquíni que é considerado a primeira peça ready to wear completamente impressa em 3D, sem necessidade de costura. A peça foi nomeada N12 bikini, por ser fabricada com nylon 12, um material sólido que pode ser dobrado sem quebrar (Continuum,2017; Bastos, 2014).

Em 2015, a designer israelense Danit Peleg chamou a atenção por seu trabalho de conclusão do curso, no qual desenvolveu cinco modelos impressos em 3D utilizando um material maleável chamado FilaFlex. O relato da designer mostra que a impressão 3D de roupas ainda não é viável: foram necessárias de 100 a 500 horas para imprimir cada peça, o que encareceu os produtos. Ademais, o material "têxtil" foi impresso no tamanho de uma página A4, sendo necessário unir as partes manualmente com uma cola especial para a construção das roupas (Kresch, 2015).

Esse relato corrobora a opinião de Gershenfeld (2012), o qual declarou que, apesar da fama das impressoras 3D, são outras tecnologias de fabricação digital as responsáveis, atualmente, pela produção da maioria dos projetos realizados em makerspaces. Duas das razões apontadas pelo autor, e demonstradas pelo trabalho de Peleg, são 1) o fato de a impressão ser lenta e 2) o limite de tamanho da mesa de impressão, enquanto outras máquinas CNC são capazes de produzir mais rapidamente e permitem o desenvolvimento de produtos maiores, mais fortes ou com detalhes mais finos.

Apesar dessas limitações, Danit Peleg já desenvolveu outras peças impressas em 3D e lançou seu próprio site, no qual é possível personalizar e encomendar uma jaqueta (Peleg, 2017). Na figura 2, são apresentadas as duas coleções desenvolvidas pela designer. $\mathrm{Na}$ imagem, é possível observar que a impressão 3D apresenta a possibilidade de desenvolver diversas superfícies para o vestuário, algumas das quais provavelmente seriam difíceis de se obter a partir de 
materiais têxteis convencionais, enquanto outras remetem a construções têxteis mais triviais, como tricô ou renda.
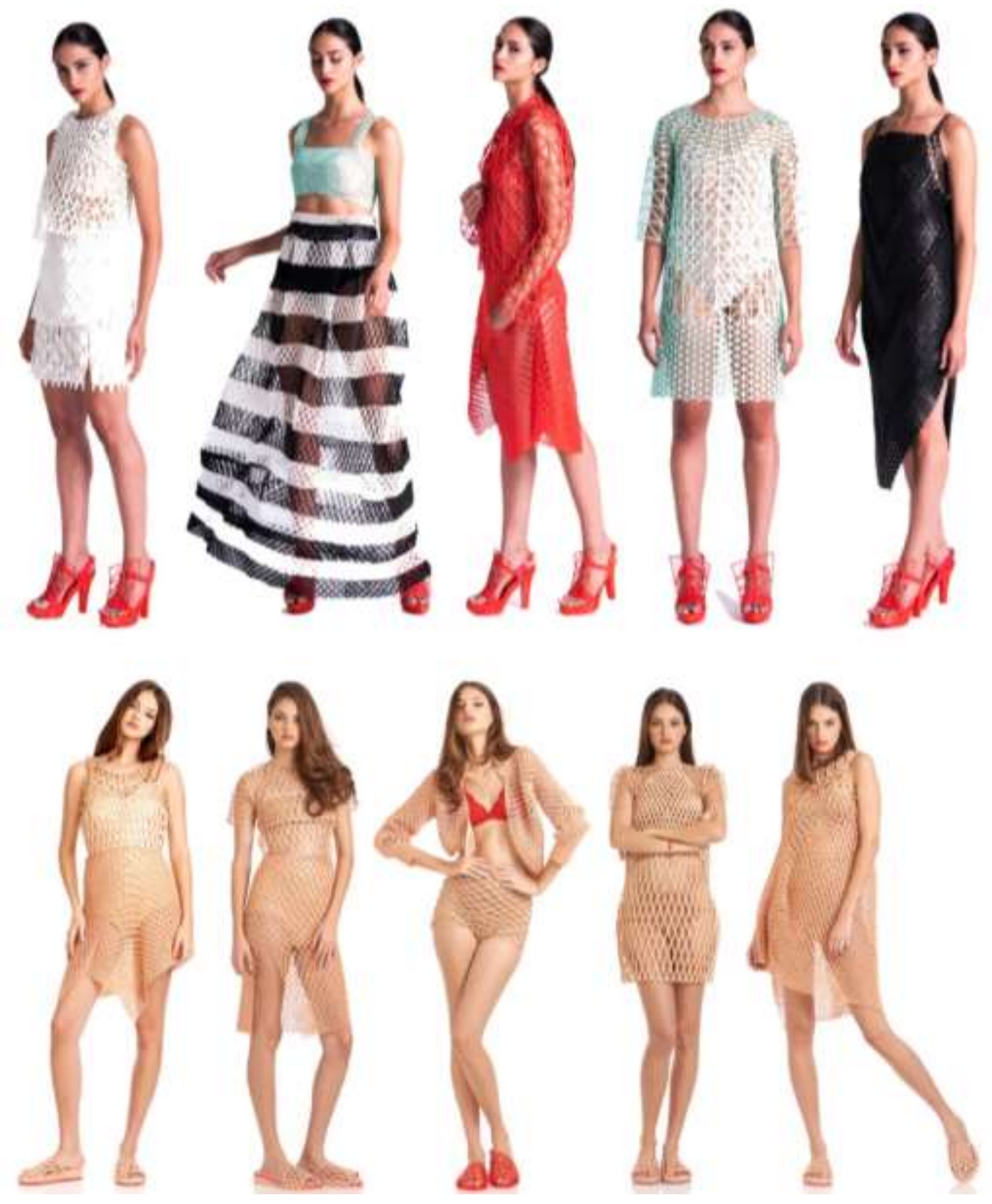

Figura 2: Modelos impressos em 3D desenvolvidos por Danit Peleg (Adaptado de peleg, 2017).

Como já mencionado anteriormente, a impressão 3D ainda apresenta algumas limitações a serem superadas, sobretudo para a construção de vestuário. Segundo Velden (2016), há restrições quanto aos materiais, pois poucos filamentos para impressoras 3D apresentam potencial para a produção de roupas confortáveis. A pesquisadora aponta a flexibilidade do filamento e do material impresso como 0 maior desafio, atualmente, para a utilização de impressoras 3D no setor de vestuário. 
Enquanto essas questões não são resolvidas, é possível utilizar a impressão 3D para criar estampas e texturas sobre o tecido, como mostram os testes do projeto Fab Textile, do Fab Lab Barcelona (Figura 3). Segundo o site do projeto, foram realizados testes bem sucedidos com filamentos flexíveis e tecidos em viscose e lycra, enquanto tecidos sintéticos com superfície lisa ou malhas com trama mais aberta não apresentaram boa adesão (Fab Textile, 2016a,b).
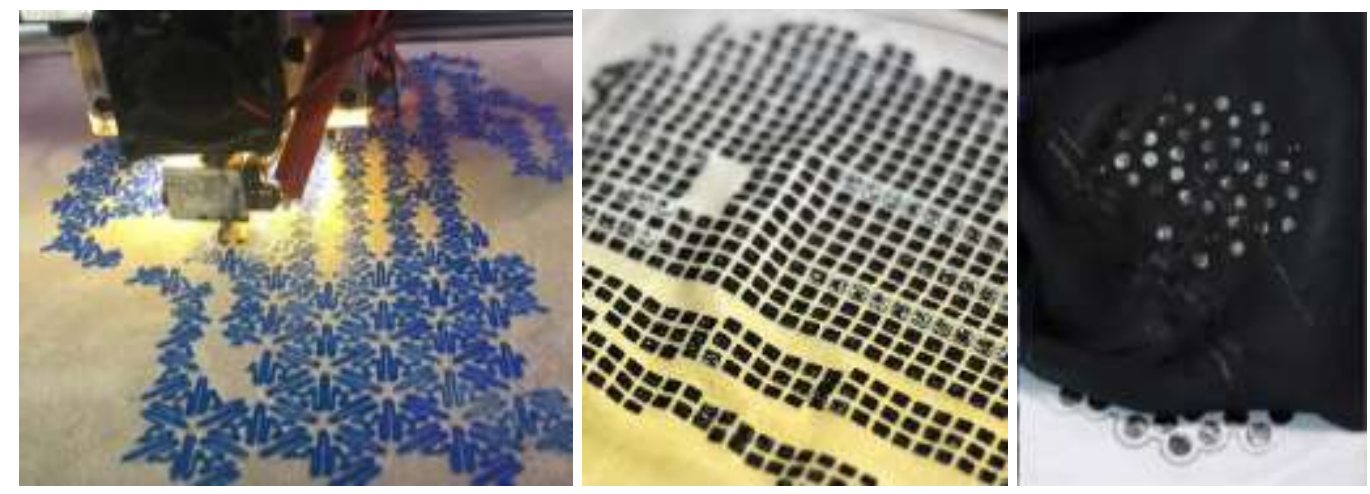

Figura 3: Testes de impressão 3D sobre tecido (Adaptado de fab textile, 2016A,B)

Para quem deseja realizar testes de impressão 3D sobre têxteis ou de materiais similares ao tecido, é possível fazer o download de arquivos CAD de diferentes desenhos e tipos de "malhas" desenvolvidas e disponibilizadas por membros das comunidades destinadas à criação e ao compartilhamento de arquivos para impressão 3D, como o Thingiverse, apontado por Abel, Evers e Klaassen (2011).

\subsection{Máquinas de tecelagem digital}

A tecelagem digital pode ser considerada uma tecnologia aditiva que utiliza fios comumente empregados na fabricação de vestuário, baseando-se em técnicas convencionais (Niinimäki; Hassi, 2011; Atwell, 2014; Velden, 2016). A tecelagem, segundo Pezzolo (2007), é um processo que se destina à produção tanto de tecidos planos, quanto de malhas e de tecidos do tipo laçada, havendo diferenças com relação ao maquinário utilizado e ao modo de tecer os fios.

A maioria dos exemplos de uso da tecelagem digital para a produção personalizada e em pequena escala encontrados na literatura, no entanto, referemse à construção de produtos de malha. Isso se reflete, também, nos nomes 
adotados para descrever os novos equipamentos digitais desenvolvidos com esta finalidade. Enquanto Niinimäki e Hassi (2011) utilizam o termo "tecelagem digital", outros autores utilizam expressões como: máquinas digitais de tricô ${ }^{8}$ (Velden, 2016; Kniterate, 2017), impressora de fiação de fios $^{9}$ (Atwell, 2014) e tricô 3D (Cunha, 2017b).

Empresas de tecelagem modernas já utilizam teares de controle computadorizado (Pezzolo; 2007). Para a construção de malha, por exemplo, são utilizadas as máquinas eletrônicas Stoll, Shima Seiki e Protti, indicadas por Udale (2009). Como explica a autora supracitada, máquinas digitais de malharia permitem a produção de tecidos com complexos tratamentos de superfície, sendo que o uso de sistemas de CAD "permite que os designs de malharia sejam rapidamente alterados para responder às tendências de moda" (Ibidem, p. 82).

Dentre as soluções industriais, destaca-se, aqui, a tecnologia whole garment, introduzida pela empresa japonesa Shima Seiki em 1995, que permite a construção, em malharia tubular, de peças inteiras, sem costura, tecidas já na forma tridimensional do corpo humano (Shima Seiki, 2017; Sissons, 2012). O uso desse tipo de tecnologia apresenta grande potencial para a fabricação pessoal por dispensar o processo de costura da peça, possibilitando que mesmo pessoas sem essa habilidade produzam suas próprias roupas.

Contudo, as máquinas digitais utilizadas pela indústria de moda foram projetadas para a produção industrial em larga escala, sendo, no geral, inadequadas para uso pessoal (Velden, 2016). Atualmente, novas máquinas tem sido desenvolvidas com este intuito. Uma delas é a Knitic, uma versão eletrônica das antigas máquinas manuais de tricô. Com ela, é possível criar digitalmente padronagens exclusivas, mas há limitações com relação ao tamanho e ao peso da máquina, que não é capaz de tricotar peças já completas, sem costura (Ibidem).

Outra tecnologia criada para atender à comunidade maker é a OpenKnit, desenvolvida por Gerard Rubio como um equipamento open hardware capaz de produzir peças inteiras (Atwell, 2014; Bastos, 2014; Velden, 2016). A máquina, no entanto, não produz padrões complexos e trabalha com no máximo três cores de linha de uma vez. Seus produtos não apresentam qualidade profissional e a 
máquina não é totalmente automática, pois algumas partes do processo requerem intervenção humana (Atwell, 2014; Velden, 2016).

Para solucionar estas questões, o criador da OpenKnit desenvolveu, no início de 2017, um novo equipamento - ainda em fase de financiamento coletivo -, a Kniterate. Destinado para a produção em pequena escala de peças inteiras, 0 dispositivo é um pouco maior que uma impressora de papel caseira e permite o uso de mais de seis cores de linhas, criando padrões, estruturas e formas que seriam difíceis de tricotar de outra maneira (Kniterate, 2017).

Enquanto equipamentos de uso pessoal ainda estão em desenvolvimento, empresas de moda tem explorado as máquinas industriais para a criação de minifábricas de roupas personalizadas. A Unmade é uma delas. Fundada em 2013, a marca oferece a oportunidade de personalizar as cores e padronagens de suas peças de tricô por meio de um aplicativo na internet. As roupas são produzidas por encomenda online ou nas lojas-fábrica da empresa (Cunha, 2015).

A marca esportiva Adidas também tem investido neste segmento. No primeiro trimestre de 2017, montou uma "loja fábrica pop-up", chamada Knit for You, no Shopping Bikini, em Berlim, onde os clientes podiam produzir, na hora, suéteres de tricô personalizados. As roupas eram produzidas nas medidas dos usuários, cujo corpo era escaneado, e personalizadas de acordo com suas especificações, por meio de softwares que auxiliam a customização das padronagens (Cunha, 2017b).

\subsection{Bordadeira digital}

A máquina de bordado digital, capaz de bordar sobre qualquer material têxtil a partir de desenhos vetoriais, já é utilizada pela indústria têxtil e de vestuário, mas é pouco presente no contexto dos makerspaces (Bastos, 2014). Uma exceção é - Fab Lab Barcelona, que possui este maquinário e o utiliza para diversos testes, inclusive para bordar com linhas que conduzem eletricidade (Eychenne; Neves, 2013; Bastos, 2014). No Fab Lab Amsterdam, também é utilizado o bordado digital, 
mas em combinação com materiais inusitados, como madeira e acrílico (Bastos, 2014).

No projeto de pesquisa Make/Use, coordenado pela designer Holly McQuillan na Massey University, Nova Zelândia, o bordado digital é explorado como recurso para simplificar a fabricação de vestuário do tipo "faça-você-mesmo". A tecnologia é utilizada como instrução, acabamento e alternativa para aumentar as possibilidades de personalização do produto (Mcquillan, 2016). O bordado é empregado principalmente nas áreas de corte, facilitando a identificação das linhas que devem ser cortadas e o acabamento da peça (Figura 4), bastando passar uma costura reta feita à máquina ou manualmente para que o tecido não desfie, dispensando a necessidade de costurar a barra (lbidem).

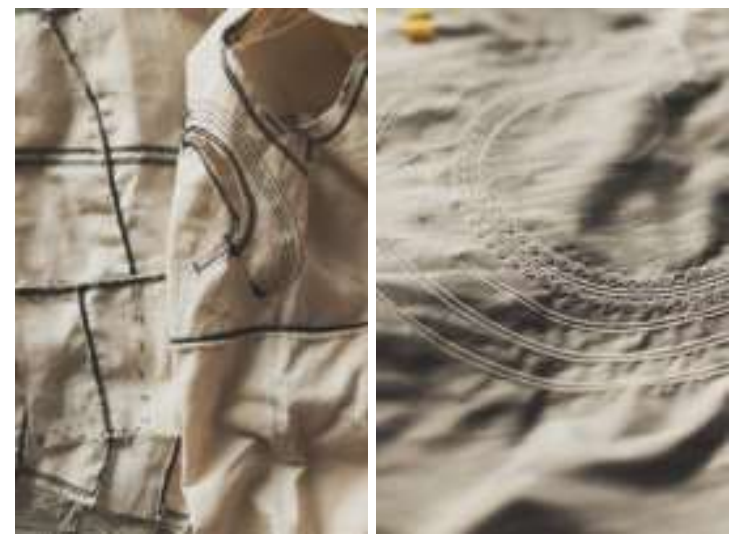

Figura 4: Bordados desenvolvidos no projeto Make/Use (Adaptado de make/use, 2017)

McQuillan (2016), no entanto, relata que a utilização do bordado digital proposta pelo projeto ainda precisa de mais exploração, pois foram encontradas dificuldades técnicas com relação ao tamanho de mesa da máquina e à facilidade de colocação em relação à estamparia digital, além do impacto da aplicação do bordado sobre o peso e o caimento do tecido.

\subsection{Máquinas de estamparia digital}

A tecnologia digital para estamparia têxtil foi desenvolvida em meados da década de 1990 e aperfeiçoada no início deste século (Cunha, 2011; Neira, 2012; Guimarães, 2014; Souza; Reis, 2014). Atualmente, as duas tecnologias de estamparia digital mais utilizadas são 1) impressão por jato de tinta, que imprime a 
imagem diretamente sobre o tecido, e 2) sublimação digital, ou impressão indireta por transferência de calor (Carvalho; Rüthschilling, 2016; Carvalho, 2015).

Alguns fatores determinam a escolha entre as tecnologias de estamparia digital. Um deles é a composição do tecido a ser utilizado. A impressão a jato de tinta é mais adequada para fibras naturais, como algodão, enquanto a sublimação digital destina-se às fibras sintéticas, como poliéster, sendo necessário que o tecido apresente no mínimo $50 \%$ de fibra sintética em sua composição para garantir a qualidade da estampa e solidez das cores (Carvalho; Rüthschilling, 2016). Outro coeficiente para seleção é a etapa da cadeia produtiva em que será aplicada a estampa. A impressão a jato de tinta é adequada apenas para estampar o tecido plano antes da etapa de confecção, pois a impressora não comporta o produto já confeccionado. A sublimação digital, por sua vez, permite estampar peças prontas (Laschuk; Rüthschilling, 2015).

Com relação à serigrafia, técnica de estamparia têxtil mais empregada pela indústria de moda atualmente, os autores consultados indicam que a estamparia digital apresenta inúmeras vantagens (Cunha, 2011; Neira, 2012; Guimarães, 2014; Souza; Reis, 2014). No presente artigo, destaca-se a eliminação de processos relacionados à gravação de matriz, apontada por Guimarães (2014) e Neira (2012). Este fator reduz também custos, motivo pelo qual a estamparia digital é, geralmente, mais adequada do que a serigrafia para a realização de pequenas tiragens (Carvalho; Rüthschilling, 2016; Carvalho, 2015). É também mais apropriada, portanto, para a personalização de produtos.

Um potencial uso da estamparia digital em um contexto de produção distribuída é apresentado por Rissanen e McQuillan (2016) e McQuillan (2016): aplicando estampas que auxiliem o usuário a compreender como montar sua própria peça. A designer neozelandesa Julia Lumsden, por exemplo, desenvolveu uma camisa que utiliza estamparia digital para indicar onde cada parte da roupa se conecta com outra no processo de construção da camisa. Como pode ser visualizado na Figura 5, diferentes cores são utilizadas nas extremidades de cada parte da modelagem, as quais são incorporadas no produto final. Além disso, marcações de costura (linhas vermelhas mais finas) foram também aplicadas na 
estampa com a finalidade de auxiliar o usuário a confeccionar o produto (Mcquillan, 2016; Rissanen; Mcquillan, 2016).
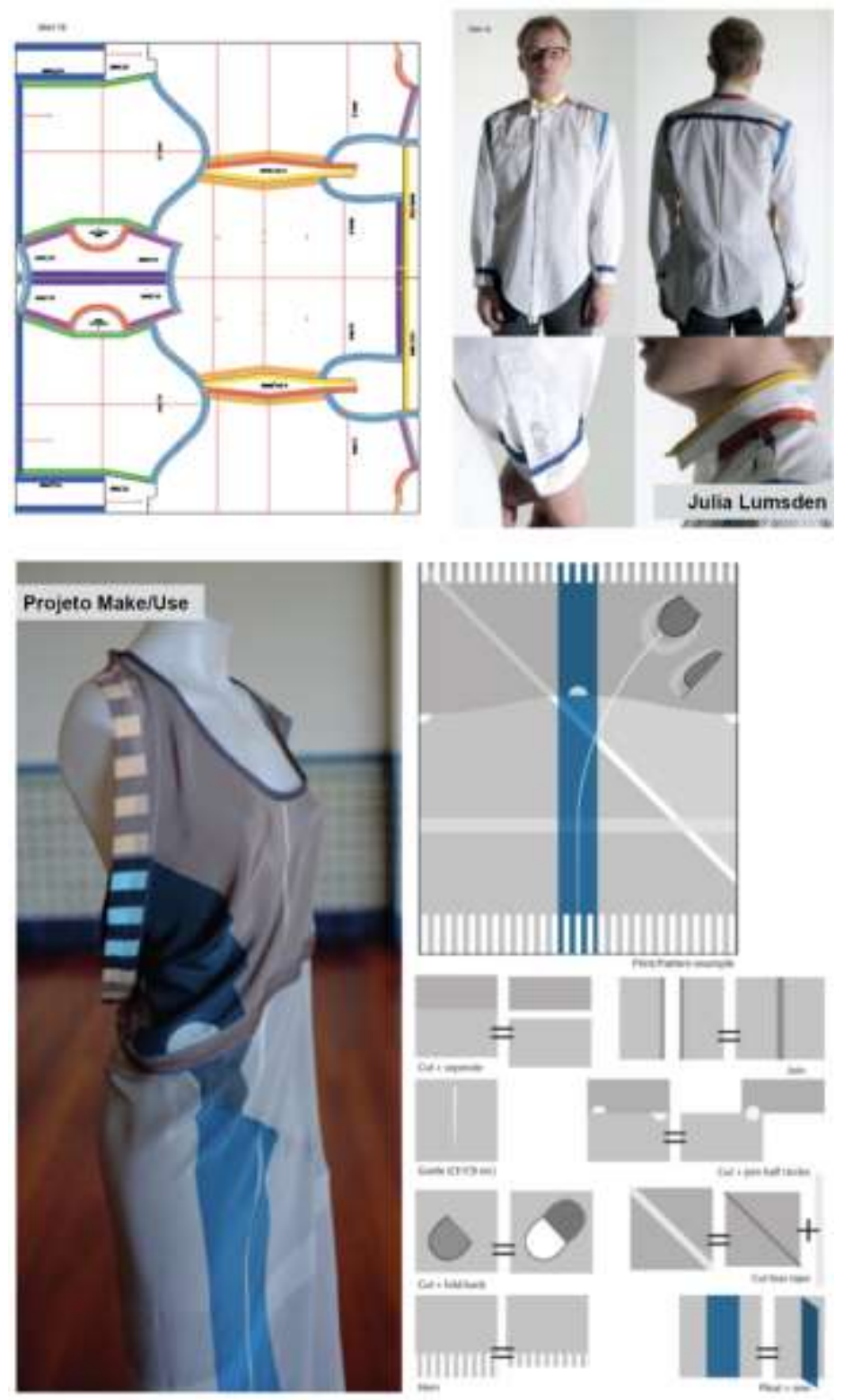

Figura 5: uso da estamparia digital para incorporar instruções destinadas à fabricação pessoal (adaptado de Mcquillan, 2016, p. 9 e 11)

Outro exemplo é o vestido desenvolvido no projeto de pesquisa Make/Use, coordenado pela designer e pesquisadora Holly McQuillan na Massey University, Nova Zelândia. Inspirada pelo trabalho de Julia Lumsden, McQuillan elaborou uma estampa que, acompanhada de um sistema de mapeamento visual (Figura 5), pudesse auxiliar na confecção do vestuário e nas modificações subsequentes, assim como resultar em uma estética visual única (Mcquillan, 2016). 
Para a designer, aplicar instruções sobre o próprio vestuário pode facilitar a transformação do tecido bidimensional em vestuário tridimensional.

Atualmente, a estamparia digital ainda apresenta algumas restrições com relação a custo do maquinário, disponibilidade de insumos fabricados no país, impressão de efeitos especiais (como gel, metalizado e flocado), velocidade de impressão e composição dos tecidos que podem ser utilizados (Neira, 2012; Guimarães, 2014; Carvalho, 2015). No entanto, Cunha (2011) mostra que já estão sendo desenvolvidas máquinas capazes de superar essas barreiras. Quanto ao seu uso para a fabricação pessoal, Bastos (2014) aponta como entrave o fato de não ser uma tecnologia presente, atualmente, na maioria dos makerspaces.

\subsection{Cortadora a laser}

As cortadoras a laser são uma das principais tecnologias de fabricação digital. São ideias para iniciantes, pois são relativamente rápidas, simples, seguras e podem ser utilizadas a partir de qualquer software de desenho vetorial (Anderson, 2012; Neves, 2014). Trata-se de uma máquina de comando numérico (CNC) que direciona com precisão um feixe de laser sobre o material a ser cortado ou gravado, movimentando-se em dois eixos. É a potência do laser que define a espessura dos materiais a serem cortados, estando relacionada, também, com a velocidade de operação da máquina (Neves, 2014).

No setor de vestuário, é utilizada principalmente para o corte de tecido ou couro, mas não permite cortar enfesto, pois a potência do laser teria de ser muito alta, o que queimaria o tecido a ser cortado (Oliveira, 2013). A cortadora a laser já tem sido utilizada industrialmente sobretudo para fazer acabamentos em tecidos e para o corte de lingerie sem costura (Oliveira, 2013; Bastos, 2014).

No contexto da fabricação pessoal, o corte a laser tem sido empregado para o recorte de estruturas de encaixe que dispensam costura a máquina e criam diferentes padrões e estruturas. Um exemplo é o projeto desenvolvido pela arquiteta Anastasia Pistofidou, do Fab Lab Barcelona, que utilizou o corte a laser para desenvolver cinco roupas DIY sem costura (Figura 6). Segundo a arquiteta, os materiais mais adequados para essas roupas são os tecidos que não desfiam e que 
"ficam no lugar", como malha neoprene e couro. Antes de desenvolver a roupa, ela recomenda criar o padrão de encaixe que substituirá a costura (Cunha, 2017c).
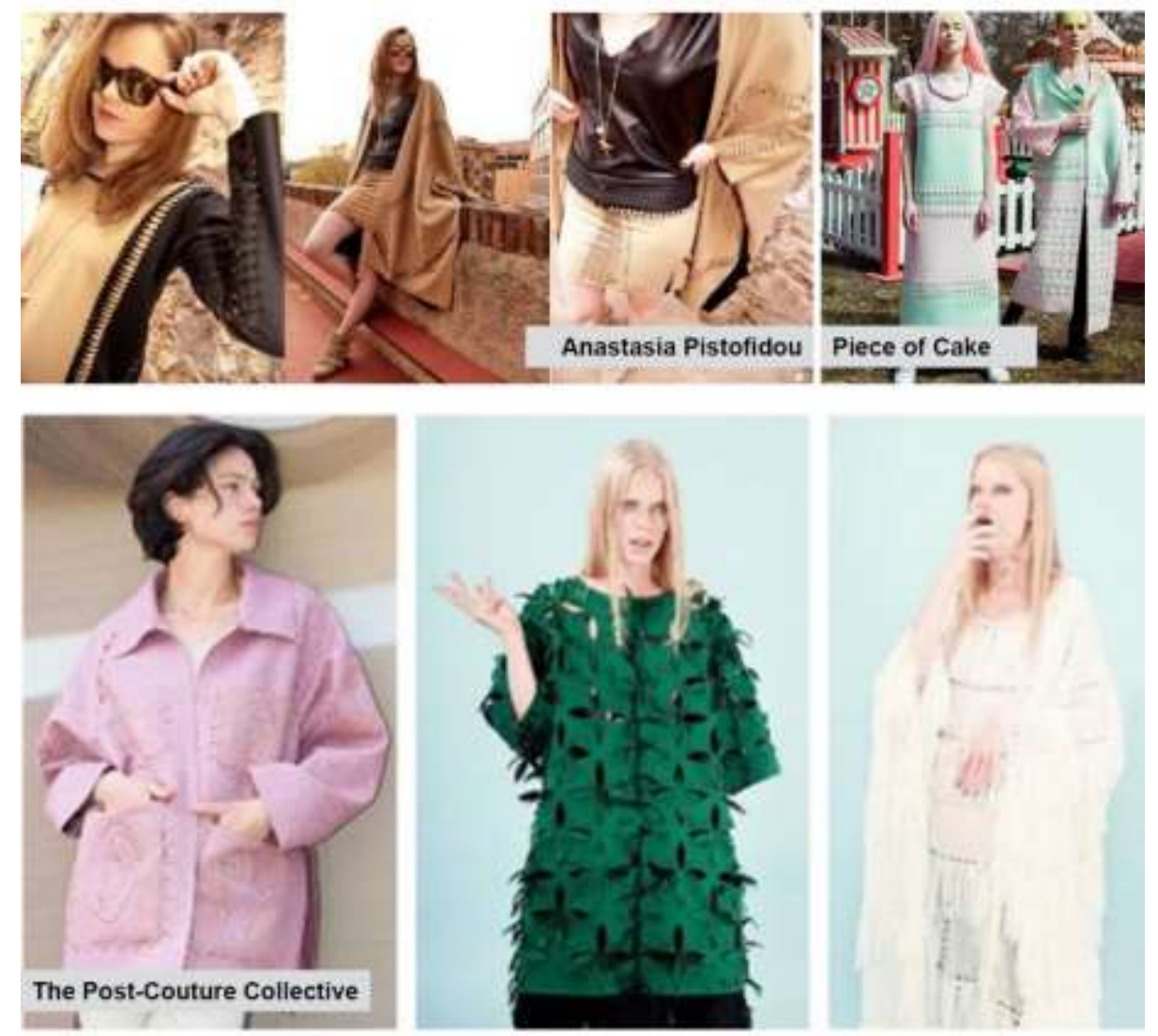

Figura 6: Produtos DIY cortados a laser (adaptado de Cunha, 2017c; Piece of Cake, 2017; The Post-Couture Collective, 2017)

O uso do laser para o recorte de estruturas de encaixe foi igualmente explorado pela designer Krista Tulp durante o desenvolvimento de sua coleção de mestrado, nomeada Piece of Cake (2017), que é também apresentada na imagem anterior. Outro exemplo de utilização do corte a laser nesse sentido é o empreendimento holandês The Post-Couture Collective, que desenvolve vestuário para a fabricação pessoal, comercializado na forma de kits de construção e de moldes digitais (The Post-Couture Collective). Para a produção das peças da primeira coleção, lançada em outubro de 2015, o laser foi empregado apenas para cortar o tecido e os conectores utilizados em substituição às máquinas de costura, como nos exemplos anteriormente mostrados. Nas últimas coleções, no entanto, a tecnologia tem sido mais amplamente explorada pela marca para a criação de intervenções têxteis, seja por meio de gravação de imagens (como nos bolsos do 
casaco cor de rosa da Figura 6), de cortes decorativos (casaco verde) ou que permitam a construção artesanal por meio de amarração de fitas (vestido branco).

O corte a laser também pode ser utilizado para a criação de estampas. Segundo Bastos (2014), o Fab Lab Barcelona realizou testes para a criação de imagens a partir de pequenos furos cortados a laser. A autora também indica o trabalho do designer coreano Eunsuk Hur, que cria impressão em têxteis, gravuras e compostos multicamadas por meio do corte a laser (Ibidem).

Aplicações semelhantes do laser foram investigadas pela designer inglesa Kate Goldsworthy durante seu doutorado. A pesquisadora explorou essa tecnologia para o beneficiamento de tecidos de poliéster, tendo como objetivo criar produtos monomateriais que possam ser mais facilmente reciclados (Goldsworthy, 2009; Gwilt, 2014). Segundo Goldsworthy (2009), quando aplicado em camadas únicas de tecidos finos, o laser cria efeitos de transparência. Sobre tecidos grossos, resulta em um efeito de superfície fundida, semelhante à laminação por pontos ou revestimento, o que levou à exploração de aplicação de aviamentos e outros materiais sobre o tecido. Também foram obtidos resultados satisfatórios com a criação de compostos multicamadas. Outra abordagem explorada foi a aplicação do laser para criar efeitos como os de malhas jacquard e gravação em relevo. Ao todo, foram testados 20 processos (lbidem).

Akiwowo et al. (2014) também investigaram a aplicação do laser sobre tecidos de poliéster, mas com foco na técnica de "tingimento a laser digital"10, a qual permite a coloração do tecido e a criação de padronagens. Já a designer alemã Elisa Strozyk explorou o corte a laser de pedaços de madeira que, ao serem aplicados sobre tecido, criam composições de padronagem geométrica que dão origem a diferentes texturas e efeitos tridimensionais (Bastos, 2014).

\subsection{Outras tecnologias: cortadora de vinil e fresadora CNC}

Duas tecnologias ainda pouco exploradas no campo da moda, mas muito presentes em makerspaces, são apresentadas por Bastos (2014) como alternativas para o Design de Superfície: a cortadora de vinil e a fresadora CNC. Segundo Neves (2014, p. 140), a cortadora de vinil atua basicamente como uma impressora 
caseira de papel que, "ao invés de reservatórios de tinta, possui em sua cabeça de impressão uma fina lâmina de aço". Com ela, é possível cortar vinil e alguns tipos de tecido, dentre outros materiais (Bastos, 2014; Neves, 2014). Foi realizado um teste no Fab Lab Groningen (Holanda) com este equipamento, segundo relato de Bastos (2014), para estampar uma camiseta: o desenho de um navio foi recortado em vinil e colado ao tecido por meio de prensa térmica.

Bastos (2014) aponta como um dos projetos mais interessantes, no entanto, o realizado com uma Fresadora CNC no Fab Lab Zürich. A fresadora é "uma máquina por comando numérico dotada de uma fresa em sua cabeça que se move sobre três eixos ( $\mathrm{X}, \mathrm{Y}$ e $\mathrm{Z}$ ). A fresa desbasta o material, retirando parte dele segundo o desenho que lhe foi enviado" (Eychenne; Neves, 2013, p. 30). No experimento do Fab Lab Zürich, a fresa da máquina foi substituída por uma caneta colorida, que transferiu para o tecido o desenho realizado em um software vetorial.

\section{Considerações finais}

As tecnologias de fabricação digital são consideradas inovadoras e apresentam o potencial de mudar a relação entre designers, produtores e usuários, uma vez que favorecem a fabricação pessoal ou em pequena escala e utilizam, para a produção, arquivos digitais que podem ser facilmente compartilhados pela internet. No caso do Design de Superfície em produtos de moda, as tecnologias relatadas no presente artigo apresentam inúmeras possibilidades de uso, permitindo que designers de superfície explorem com mais liberdade sua criatividade e convidem o usuário a participar do processo de criação ou customização das superfícies têxteis. Também é possível utilizar o Design de Superfície para criar sobre o tecido, por meio de tecnologias de fabricação digital como o bordado ou a estamparia digital, intervenções que auxiliem o usuário a produzir sua própria peça.

As tecnologias de fabricação digital, no entanto, ainda estão em fase de desenvolvimento e popularização, sobretudo na indústria de moda. Como revelam os resultados da revisão bibliográfica sistemática conduzida pelos presentes autores, poucas publicações exploram a aplicação de tecnologias de fabricação digital em produtos de moda. A pesquisa nesta área ainda se encontra, portanto, em estágio inicial. Assim como o desenvolvimento de equipamentos apropriados à 
indústria de moda: algumas das tecnologias de fabricação digital precisam ser aprimoradas para tornarem-se viáveis para a produção de vestuário, enquanto outras já utilizadas na indústria de moda ainda estão sendo adaptadas para o contexto da produção distribuída e da fabricação pessoal e necessitam ser incorporadas por makerspaces.

Por essas questões, é necessário prosseguir com as investigações sobre as possibilidades apresentadas pela fabricação digital para o Design de Superfície de produtos de moda. Não obstante, considerando a velocidade com que inovações são empreendidas na área de tecnologia, seja com o desenvolvimento de novos equipamentos, seja com atualização ou mesmo obsolescência dos atuais, a pesquisa na área de fabricação digital pode ser complexa, exigindo do pesquisador constante atualização e cuidado quanto à abordagem para disseminação do conhecimento, para que este não se torne defasado em curto ou médio prazo.

\section{Agradecimentos}

Os autores agradecem à agência de fomento brasileira Capes pelo apoio financeiro concedido.

\section{Notas}

${ }^{1}$ Computer-Aided Design

${ }^{2}$ Projetos de hardware eletrônico desenvolvidos e ofertados segundo os mesmos princípios do movimento open source software.

${ }^{3}$ Associação Brasileira da Indústria Têxtil e de Confecção.

${ }^{4}$ Agência Brasileira de Desenvolvimento Industrial.

${ }^{5}$ Centro de Tecnologia da Indústria Química e Têxtil.

${ }_{7}^{6}$ Disponível em: <http://bdtd.ibict.br/vufind/>.

${ }^{7}$ Digital weaving machines, no original.

${ }^{8}$ Digital knitting machine, no original.

${ }^{9}$ Yarn-weaving printer, no original.

${ }^{10}$ Digital Laser-dyeing, no original. 


\section{Referências}

ABDI; FUNDAÇÃO CERTI. Conceituação da Empresa de Confecção do Futuro Florianópolis: 2015. Relatório técnico. Disponível em: <http://www.abdi.com.br/Estudo/Conceituação da Empresa de Confecção do Futuro.pdf>. Acesso em: 20 fev. 2017.

ABEL, B. van; EVERS, L.; KLAASSEN, R. Preface. In: ABEL, B. van Abel; Klaassen, R.; Evers, L.; Troxler, P. (Eds.). Open Design Now: Why Design Cannot Remain Exclusive. Amsterdam: BIS publishers, 2011. Disponível em: <http://opendesignnow.org/index.html?p=38.html>. Acesso em: 19 nov. 2016.

AKIWOWO, K. et al. Digital Laser-dyeing for Polyester Fabrics. Journal of Textile Design Research and Practice, v. 2, n. 2, p. 133-151, 2014.

ANDERSON, Chris. Makers: a nova revolução industrial. Tradução de: SERRA, A. C. C. Rio de Janeiro: Elsevier, 2012. Título original: Makers - the new industrial revolution.

ATWELL, C. Yarn-weaving printer creates custom clothes: Will a new open-source DIY knitting machine from OpenKnit change the way we make clothes? Design News, v. 69, n. 6, p. 30-31, 2014.

BALKA, K.; RAASCH, C.; HERSTATT, C. Open source enters the world of atoms: a statistical analysis of open design. First Monday, v. 14, n. 11, 2009.

BASTOS, V. F. Moda e fabricação digital em um contexto Fab Lab: equipamentos, métodos e processos para o desenvolvimento de produtos. $151 \mathrm{f}$. Dissertação (Mestrado em Design) - Centro de Artes e Comunicação, Universidade Federal de Pernambuco, Recife, 2014.

BAUWENS, M. et al. Synthetic overview of the collaborative economy. Amsterdam: P2P Foundation, 2012. Relatório técnico.

BRUNO, FS. A Quarta Revolução Industrial do Setor Têxtil e de Confecção: a Visão de Futuro para 2030. São Paulo: Estação das Letras e Cores, 2016.

BRUNO, FS; PIMENTEL, F. Confecção 4.0: uma visão para o futuro do setor têxtil e de confecção Brasileiro. In: MENDES, F. D. (Org.). Educação de moda para o futuro: desenvolvimento sustentável nas dimensões social, econômica, ambiental, cultural e geográfica. São Paulo: $\mathrm{EACH} / \mathrm{USP}$, 2017. p. 131-137.

CARVALHO, NA. Estudo comparativo de processos de estamparia têxtil sob enfoque ambiental: uma contribuição para o design de moda. 115 f. Dissertação (Mestrado em Design) Faculdade de Arquitetura, Universidade Federal do Rio Grande do Sul, Porto Alegre, 2015.

CARVALHO, NA; RÜTHSCHILLING, E. A. Inovação em metodologia de projeto aplicada ao design de superfície voltado para moda. Modapalavra E-periódico, Florianópolis, v. 9, n. 17, p. 178-194, jan./jun. 2016.

CBD - CENTRO BRASIL DE DESIGN. Diagnóstico do Design Brasileiro. Brasília: CBD;

ApexBrasil; Ministério do Desenvolvimento, Indústria e Comércio Exterior, 2014. Relatório técnico.

CONFORTO, E. C.; AMARAL, D. C.; SILVA, S. L. DA. Roteiro para revisão

bibliográficasistemática: aplicação no desenvolvimento de produtos e gerenciamento de projetos. Trabalho apresentado no $8^{\circ}$ Congresso Brasileiro de Gestão de Desenvolvimento de Produto, Porto Alegre, 2011.

CONTINUUM. N12. Disponível em: <http://www.continuumfashion.com/N12.php>. Acesso em: 01 out. 2017.

CUNHA, R. T. da. ITMA 2011: Destaques da Estamparia Digital. Redige, Rio de Janeiro, v. 2, n. 3, p. 77-86, dez. 2011. 
CUNHA, R. Unmade mostra como a customização em massa é o futuro da moda. 2015. Disponível em: <http://www.stylourbano.com.br/unmade-mostra-como-customizacao-em-massa-e-ofuturo-da-moda/>. Acesso em: 06 mar. 2017.

CUNHA, R. Em 2030, as fábricas de vestuário serão como fábricas automotivas devido a automação industrial. 2017. Disponível em: <http://www.stylourbano.com.br/em-2030-as-fabricasde-vestuario-serao-como-as-fabricas-automotivas/>. Acesso em: 03 mar. 2017.

CUNHA, R. KNIT FOR YOU - A "loja fábrica pop-up" da Adidas que produz suéteres personalizados pelo cliente. 2017. Disponível em: <http://www.stylourbano.com.br/knit-for-you-a-loja-fabrica-pop-upda-adidas-que-produz-sueteres-personalizados-pelo-cliente/>. Acesso em: 02 mar. 2017.

CUNHA, R. Arquiteta Anastasia Pistofidou cria roupas sem costura através de fabricação digital. 2017. Disponível em: <http://www.stylourbano.com.br/arquiteta-anastasia-pistofidou-criaroupas-sem-costura-atraves-de-fabricacao-digital/>. Acesso em: 03 mar. 2017.

DRESCH, Aline; LACERDA, Daniel Pacheco; ANTUNES JÚNIOR, José Antônio Valle. Design Science research: método de pesquisa para avanço da ciência e tecnologia. Porto Alegre: Bookman, 2015.

EYCHENNE, F.; NEVES, E. Fab Lab: a Vanguarda da Nova Revolucao Industrial. São Paulo: Editorial Fab Lab Brasil, 2013.

FAB TEXTILES. What. 2017. Disponível em: <http://fabtextiles.org/what/>. Acesso em: 06 mar. 2017.

FAB TEXTILES. 3D Print On Fabric - Ftex Workshop Feb 2016. 2016. Disponível em: <http://fabtextiles.org/fabtextiles-workshop-3d-print-on-fabric/>. Acesso em: 07 mar. 2017.

FAB TEXTILES. 3D Print On Fabric @Mce3 Conference. 2016. Disponível em: http://fabtextiles.org/3d-print-on-fabric/>. Acesso em: 07 mar. 2017.

GERSHENFELD, N. How to Make Almost Anything: The Digital Fabrication Revolution. Foreign Affairs, Tampa, nov.-dez. 2012. Disponível em: <https://www.foreignaffairs.com/articles/2012-0927/how-make-almost-anything>. Acesso em: 19 nov. 2016.

GOLDSWORTHY, K.; PAINE, H. Laser Welding of Textiles: a creative approach to technology through a reflective craft practice. In: INTERNATIONAL CONFERENCE PROCEEDINGS, 2014, Falmouth, Reino Unido. Disponível em <http://www.autonomatic.org.uk/allmakersnow/wpcontent/uploads/2015/07/AMN2014_Goldsworthy_et_al.pdf>. Acesso em: 19 nov. 2016.

GOLDSWORTHY, K. RESURFACED: Using Laser Technology To Create Innovative Surface Finishes For Recyclable, Synthetic Textiles. In: CUTTING EDGE: LASERS AND CREATIVE SYMPOSIUM, 2009, Loughborough, Inglaterra. Disponível em:

<https://www.researchgate.net/publication/266405877_RESURFACED_Using_Laser_Technology_To Create_Innovative_Surface_Finishes_For_Recyclable_Synthetic_Textiles>. Acesso em: 19 nov. 2016.

GUIMARÃES, F. Inovações em processos de impressão têxtil. dObra[s], São Paulo, v. 7, n. 15, p. 90-97, mar. 2014.

GWILT, Alison. Moda sustentável: um guia prático. Traduzido por: LONGARÇO, M. São Paulo: Gustavo Gili, 2014. Título original: A practical guide to sustainable fashion.

KNITERATE. Digital Knitting Machines. 2017. Disponível em: <http://www.kniterate.com/>. Acesso em: 30 ago. 2017.

KOHTALA, C. Addressing sustainability in research on distributed production: An integrated literature review. Journal of Cleaner Production, v. 106, p. 654-668, 2015. 
KOHTALA, C; HYYSALO, S. Anticipated environmental sustainability of personal fabrication. Journal of Cleaner Production, v. 99, p. 333-344, 2015.

KRESCH, Daniela. Designer israelense cria roupas em impressoras 3D. 2015. Acesso em: <http://www1.folha.uol.com.br/mercado/2015/09/1678350-designer-israelense-cria-roupas-emimpressoras-3d.shtml>. Acesso em: 07 mar. 2017.

KUHN, R.; MINUZZI, R. DE F. B. Panorama da impressão 3D no design de moda. In: MODA DOCUMENTA, 2015, 5., São Paulo. Disponível em: <http://www.modadocumenta.com.br/anais/anais/5-Moda-Documenta-2015/02-Sessao-TematicaDesign-Moda-e-Cultura-Digital/Renato-Kuhn_ModaDocumenta2015_PANORAMA-DA-IMPRESSA_O3D-NO-DESIGN-DE-MODA.pdf>. Acesso em: 26 jan. 2017.

LASCHUK, T.; RÜTHSCHILLING, E. A. Adequação dos processos de estamparia nas moda e vestuário. In: COLÓQUIO DE MODA, 11., 2015, Curitiba. Anais do Colóquio de Moda. Disponível em: <http://www.coloquiomoda.com.br/anais/anais/11-Coloquio-de-Moda_2015/ARTIGOS-DEGT/GT04-DESIGN-E-PROCESSOS-DE-PRODUCAO-EM-MODA/GT-4-ADEQUACAO-DOSPROCESSOS-DE-ESTAMPARIA.pdf>. Acesso em: 20 jan. 2017.

LeNSin - INTERNATIONAL LEARNING NETWORK ON SUSTAINABILITY. The LeNSin research hypothesis: the design of S.PSS applied to DE: win-win offer model for a sustainable development for all. Milão: Politecnico di Milano - Design dept, 2016. Relatório de pesquisa.

MAKE/USE. Tube dress. Disponível em: <https://makeuse.nz/make/tube-dress/>. Acesso em: 03 mar. 2017.

MCQUILLAN, H. MakeUse V2: digital textile technology for user modifiable zero waste fashion. In: SHAPESHIFTING: CONFERENCE ON TRANSFORMATIVE PARADIGMS OF FASHION AND TEXTILE DESIGN, 2016, Auckland, Nova Zelândia. Disponível em:

$<$ https://www.academia.edu/15522170/Make_Use_V2_digital_textile_technology_for_user_modifiable _zero_waste_fashion>. Acesso em: 19 nov. 2016.

NEIRA, L. G. Impressão digital, estética artesanal. Redige, Rio de Janeiro, v. 3, n. 1, p. 18-31, abr. 2012.

NEVES, H. Maker Innovation: do Open Design e Fab Labs ... às estratégias inspiradas no movimento Maker. $261 \mathrm{f}$. Tese (Doutorado em Arquitetura e Urbanismo) - Faculdade de Arquitetura e Urbanismo, Universidade de São Paulo, 2014.

NEVES, H; ROSSI, D. Open Design. In: CONGRESSO BRASILEIRO DE GESTÃO DE DESENVOLVIMENTO DE PRODUTO, 8, 2011, Porto Alegre.

NIINIMÄKI, K.; HASSI, L. Emerging design strategies in sustainable production and consumption of textiles and clothing. Journal of Cleaner Production, v. 19, n. 16, p. 1876-1883, 2011.

OLIVEIRA, M. C. DE. Procedimentos paramétricos aplicados ao design de moda. $104 \mathrm{f}$.

Dissertação (Mestrado em Arquitetura e Urbanismo) - Universidade Presbiteriana Mackenzie, São Paulo, 2013.

PELEG, Danit. 3D Printed Fashion \#printwear3d by Danit Peleg. 2017. Disponível em: <https://danitpeleg.com/>. Acesso em: 01 out. 2017.

PEZZOLO, Dinah Bueno. Tecidos: história, tramas, tipos e usos. São Paulo: Editora Senac São Paulo, 2007.

PIECE OF CAKE. Home. 2017. Disponível em: <http://piece-of-cake.ee/>. Acesso em: 30 ago. 2017.

RICHARDSON, M. Pre-hacked: Open Design and the democratisation of product development. New Media and Society, v. 18, n. 4, p. 653-666, 2015. 
RISSANEN, Timo; MCQUILLAN, Holly. Zero waste fashion design. Londres: Bloomsbury, 2016.

SHIMA SEIKE. About Wholegarment. 2017. Disponível em:

<http://www.shimaseiki.com/wholegarment/>. Acesso em: 01 out. 2017.

SISSONS, Juliana. Malharia. Porto Alegre: Bookman, 2012.

SOUZA, P. de M.; REIS, F. S. dos. Trompe-l'oeil em estamparia digital: um recurso para alterar a percepção da silhueta masculina. Projetica, Londrina, v. 5, n. 2, p. 69-86, dez. 2014.

STIKKER, M. Introduction. In: ABEL, B. van Abel; Klaassen, R.; Evers, L.; Troxler, P. (Eds.). Open Design Now: Why Design Cannot Remain Exclusive. Amsterdam: BIS publishers, 2011. Disponível em: < http://opendesignnow.org/index.html?p=19.html>. Acesso em: 19 nov. 2016.

STRIEN, M. Van; PONT, V. de. Open Source Fashion Manifesto. Rotterdam: Het Nieuwe Instituut, 2016.

THE POST-COUTURE COLLECTIVE. Shop. 2017. Disponível em:

<http://www.postcouture.cc/shop/>. Acesso em: 30 ago. 2017.

TROXLER, P. Libraries of the Peer Production Era. In: ABEL, B. van Abel; Klaassen, R.; Evers, L.; Troxler, P. (Eds.). Open Design Now: Why Design Cannot Remain Exclusive. Amsterdam: BIS publishers, 2011. Disponível em: <http://opendesignnow.org/index.html? $p=411 . h t m l>$. Acesso em: 19 nov. 2016.

TROXLER, P. What's next for open hardware and design? In: BRAYBROOKE, K.; NISSILA, J.; VUORIKIVI, T. (Eds.). The Open Book. Londres: The Finnish Institute in London, 2013. p. 32-39.

UDALE, Jenny. Fundamentos de design de moda: tecidos e moda. Porto Alegre: Bookman 2009.

VELDEN, N. M. van der. Making Fashion Sustainable: the role of designers. $226 \mathrm{f}$. Tese (Doutorado em Design para a Sustentabilidade) - Delft University of Technology, Haarlem, Holanda, 2016. 